\title{
COUNTERFACTUAL SUCCESS AGAIN: RESPONSE TO CARTER AND KRAMER
}

\author{
Keith Dowding and Martin van Hees
}

We would like to thank Ian Carter and Matthew Kramer for their challenging reply to our recent article. Dowding and van Hees (2007) is one of a series of articles in which we try to address measurement issues with regard to individual freedom. Our aim is to provide a conception of freedom that will eventually yield a way of measuring the relative freedom of groups of people within a society and a relative measure of freedom across societies. In doing so, we draw upon the important work of Carter (1999) and Kramer (2003), but as should be clear, we also depart from it in several respects.

\section{TEMPORAL AND COUNTERFACTUAL DEPENDENCIES}

We have argued that the "pure negative conception" of freedom embraced by Carter and Kramer fails to be what it is supposed to be: a value-neutral conception of freedom. They say that supporters of the "pure negative" conception of freedom advocate "that a person is unfree to $x$ if and only if he is prevented from $x$-ing by the conduct or dispositions of some other person(s)" (Carter and Kramer 2008: 81, we change their " $\varphi$ " to " $x$ " for consistency in our article). The definition is said to be value-neutral because preferences do not enter into either (a) the characterization of $x$ (individual $i$ 's evaluation of the worth of $x$ ); or (b) the characterization of the manner in which $x$-ing could be constrained (any way in which another person's act affects our negative freedom). In our article we argue that though preferences do not seem to enter the characterization of $x$ or of its constraints, application of the definition to a class of situations shows that an individual $i$ 's evaluation of the worth of $x$ determines $i$ 's negative freedom.

Carter and Kramer argue that pure negative theorists only need to characterize $x$-ing as not having direct preference-dependence, but can happily admit it having indirect preference-dependence. In one of our 
book examples $i$ 's freedom is dependent upon $i$ 's preferences and, since $j$ 's preferences are dependent upon $i^{\prime} \mathrm{s}, i^{\prime}$ 's freedom is also dependent upon his own preferences. Carter and Kramer make much of the first dependence not being relevant to negative liberty; it is not clear why they do so, in our account it sets up the discussion of the second dependence. Kramer and Carter object that our examples rely on the second dependence being indirect. Indirect-preference dependence is when a person's preference change causes something else in the world to change and it is that alteration in the world that affect the person's freedom. Direct preference change is when a person's preferences themselves influence his freedom. In this argument they have given up the conceptual-causal distinction that had motivated their previous defence (Kramer 2003: 190; Carter in conversation). However, it is not as clear as they imagine that their distinction is a critique of our counter-examples. The toy games by which we examine how an individual $i^{\prime}$ s preference change affects $i^{\prime} \mathrm{s}$ freedom are static games of complete information and not the dynamic games of incomplete information they seem to imagine. Their temporal interpretation not only yields different games, but different types of games. Of course, if Ami is no longer in a position to stop Fred from doing $x$ then he is free to do $x$. So what? What matters is whether our example demonstrates different ascriptions of freedom for Fred under different preferences. The correct temporal interpretation of our games is that if an agent at time $t_{0}$ with preference $x$ has freedom described by $F$ does his freedom change when his preference changes (from $x$ to $y$ ) at time $t_{0}$ ? We are discussing a counterfactual alteration of preference, not a temporal change of mind. Our argument is not aimed at elucidating the temporal dependence of freedom on desires. Indeed, such an exercise would be rather trivial.

Given that we describe a counterfactual and not a temporal change in preference, can the arguments of Carter and Kramer against a direct dependence be used against our account? That is, are they right in rejecting our claim that "one can lose a freedom simply because one's preferences have changed" (Dowding and van Hees, 2007: 21)? Carter and Kramer indeed reject this claim but they do so on the basis of a mistaken view about how to analyse counterfactuals. To see why counterfactuals are important, note that on our account as well as on Carter's and Kramer's, counterfactuals determine whether a person possesses a certain kind of freedom: for establishing whether a person is free to $x$, we must examine what happens if the person sets out or attempts to $x$. Now the actions that people take are based on a social web of beliefs, desires, intentions and information they receive from others, that constitute their and others' dispositions. How people act and interact together are based upon these webs. What people can and cannot do is often based upon what they believe they are able to do; and how they think others will react to them. A counterfactual analysis requires us to take into account these 
dispositional webs and the "can" of what people "can" do should not simply be viewed in the physicalist terms of bars, ropes and handcuffs. "Can is constitutionally iffy", and the can of negative freedom needs a careful counterfactual analysis.

To spell out the difference between our counterfactual analysis and the one (implicitly) used by Carter and Kramer in their rejection of the possibility of a direct dependence between freedom and preferences, suppose it so happens that some $i$ is not free to $x$ and has no desire to $x$. To show that there may be a preference-dependence - that is, to show that $i$ 's unfreedom depends on $i$ 's desire - we argue that for a class of situations the counterfactual statement "If $i$ were to desire $x$, he would be free to $x^{\text {" }}$ is true. Carter and Kramer now state the pure negative theorist's question as follows: "If a person's preferences have undergone some change, and if nothing else in the world has altered, can the person's freedoms have been affected by the change in her preferences?" (Carter and Kramer, 2008: 83). The mistake they make is to assume that the truth of a counterfactual is determined by the nature of the world in which, apart from the truth of the antecedent (i.e. " $i$ " desires to $x$ ) nothing else has altered. However, on the standard, Lewisian view used by us, a counterfactual is true if the consequence (" $i$ is free to $x^{\prime \prime}$ ) is true in the possible worlds that are closest to the actual world in which the antecedent is true. Given the dispositional webs described in our toy examples, the nearest possible world in which $i$ 's preferences have changed are, by assumption, worlds in which the actions of the others have changed as well, and that change results in the loss of the freedom in question. It is on the basis of this standard view about the nature of counterfactual analysis that we can indeed say that "one can lose a freedom simply because one's preferences have changed".

Once it is realized that the temporal interpretation of our analysis is inappropriate, it can quickly be seen that the arguments that Carter and Kramer put forward to defend the unavoidability and the unobjectionability of such a temporal (i.e. indirect) preference-dependence has no bearing on our analysis. But will the arguments also hold under the right, counterfactual interpretation of the dependence that we established?

Consider, first, Carter's and Kramer's claim that our intentionbased adaptation of negative freedom is also vulnerable to preferencedependencies. Now, contrary to what Carter and Kramer claim (84), we did not attempt to formulate a conception of freedom that avoids preference-dependencies altogether. As we made clear (158), we believe that our adaptation of the negative freedom conception will reduce rather than prevent the occurrence of such dependencies. However, though the example provided by Carter and Kramer thus forms a counterexample to a claim we do not make, it is interesting to examine their example in 
somewhat more detail. When analysed in terms of counterfactuals, it can be seen to be based on the above-discussed mistaken conception of the truth conditions of counterfactuals.

They discuss two variations, Games 5 and 6, of our Fred-Ami book example and conclude that under our account there is also a preferencedependence. However, the argument fails under the correct counterfactual interpretation of dependencies. To see why, consider their claim that in Game $5 \mathrm{Ami}$ will not have the intention to prevent Fred if he were to attempt to get the book. This claim is based on the assumption that, in Game 5, Ami predicts that Fred will not get the book and that Ami will therefore not try to prevent him. Now this conclusion is only warranted if, in the nearest possible world in which Fred tries to get the book, Ami will not prevent him from doing so. However, in that nearest possible world Fred will have different preferences than the ones specified in Game 5; to analyse Fred's freedom in that world we need to analyse a game in which Fred does have a desire to buy the book. Since we can assume that in nearest possible worlds the game is still one of complete information, we can expect, given Ami's primary desire of Fred not getting the book, that Ami will thwart Fred's attempt. In both games Fred is not free to read the book: there is no preference-dependence.

Next consider their argument for the irrelevance of Berlin's rejection of the concept of stoic freedom for a temporal freedom-dependence. Again we ask whether their argument can also be applied to the right interpretation of the dependence, that is, to the counterfactual one. They distinguish two scenario's. The first is the one in which a person $P$ is currently being prevented by an oppressor $Q$ to perform some set of actions but, if he would have no desire to perform the actions in question, would be said to be free even though there are no other changes in his situation (in particular, he is still prevented from performing the actions). In the second scenario the situation is the same except for the fact that the oppressor would lift the obstacles if $P$ would change his desires. Carter and Kramer argue that the change in the second situation constitutes a "real liberation" contrary to the "sham liberation" of the first situation. We concur if this is to be read as a normative statement saying that the latter change is more important rather than as an ontological argument about what freedom "really" is. However, note that the preference-dependence that we pointed out lies in-between these two situations. For those cases, it is not clear that the acquired freedom constitutes less of a "real" than a "sham" liberation. Indeed, as Carter and Kramer mention in footnote 3 of their comment, in cases in which the existence of prevention on $Q^{\prime} s$ side is conditional upon $P^{\prime}$ s desires - as is in the case in our Fred-Ami example - " $P$ 's freedom is much more precarious than it would be if $Q$ had an unconditional disposition to prevent $P$ from $x$-ing" (92, fn 3 , italics added). 


\section{FREEDOM'S NON-SPECIFIC VALUE}

To address their final remarks we need to go beyond our article itself a little and back to their original accounts of the valuation and measurement of freedom. Our response is made harder by the fact that our critics are two authors who do not have precisely the same views on these issues themselves. Carter, Kramer and ourselves agree that the non-specific value of a particular freedom (or of overall freedom) should not be related to the specific value of that particular freedom (overall freedom). ${ }^{1}$

With respect to particular freedoms, the point can be put in another (essentially equivalent) way by saying that the utility a person gains from enjoying a particular freedom, should not contribute to that particular freedom's non-specific value. Carter and Kramer believe that this rules out direct preference-dependence of the property on which the nonspecific value of freedom supervenes. Their concern is that the preference dependence will contaminate the measure of nonspecific value. However, the preference-dependence we identify and the importance of the intentions of constrainers merely affects which particular freedoms belong to a person's opportunity set. It does not affect the measurement of those freedoms in terms of their non-specific value.

Next consider overall freedom. For Carter the non-specific value of overall freedom supervenes entirely on the sheer quantity of alternatives available. He identifies that sheer quantity with overall freedom, and the non-specific value is assumed to be a (weakly monotonic) function of the levels of overall freedom. For Kramer too adding alternatives can never decrease the non-specific value of freedom, however, his measure is "partly evaluative" since additional freedoms in an opportunity set are weighted

${ }^{1}$ Carter and Kramer (2008: 16) suggest we have mistaken the specific non-specific distinction because we have ignored Kramer's tripartite classification between (1) the non-specific value of any freedom-to- $x,(2)$ the specific value of any freedom-to- $x$, and (3) the value of $x$-ing. They claim that the "way to capture the idea of freedom's non-specific value is ... through a more abstract consideration of the mere fact of having freedom - that is, through a perception of the value of freedom as such" (93). However, our example of non-specific value of freedom of expression was merely to illustrate the nature of non-specific value. Contrary to what Carter and Kramer argue in their response, it is an example of a particular freedom having non-specific value. On Carter's original definition a "phenomenon $x$ has non-specific value (...) if, and only if, the value of $x$ cannot be described wholly in terms of a good brought about or contributed by a specific instance of $x$ or set of specific instances of $x^{\prime \prime}$ (Carter, 1999: 34). To illustrate the notion thus defined, we argued that the value of having freedom of expression cannot be entirely reduced to its instances, that is, to either the actual acts of expressing one's opinions or to the various specific tokens of this freedom type. 
dependent upon the freedoms already there in order to take account of diversity considerations. ${ }^{2}$

It is not clear why non-specific value should be a (weakly) monotonic function. The non-specific value of any particular freedom might derive from a number of sources. Having freedom might instrumentally increase the welfare of society for example, and it might originate in the effects freedom has on the mental state of a person. Let us take the latter source. We might conjecture that having a large set of alternatives from which to choose fosters well-being, feelings of control, autonomy and so on. These factors (which might also be termed 'process utility') constitute a source of freedom's non-specific value.

However, continually adding alternatives to an opportunity set might not continually increase that person's process utility. Rather, as is wellknown (Schwartz, 2004) too many alternatives can have psychological costs. Some decisions are stressful, and having to make too many trivial choices could be irritating. Stated differently, some increases of one's overall freedom (sheer quantity of alternatives) will lead to a decrease in this kind of non-specific value. ${ }^{3}$ The example shows that we cannot simply assume that an increase in the extent of one's freedom can never lead to a decrease of the (net) non-specific value of that freedom. Carter and Kramer's claim that the non-specific value of freedom supervenes (positively) on the amount of overall freedom one enjoys is thus in desperate need of empirical underpinning.

Both Carter and Kramer take the pure concept of negative freedom as given and then argue that their measurement of freedom captures its nonspecific value. We think Carter's argument unconvincing since it can be doubted whether his measurement of freedom does indeed capture its nonspecific value. We think that Kramer's argument is questionable as well but for a different reason: his measurement of freedom is unconvincing as a measurement of pure negative freedom. Rather than adjusting one's measurement of freedom so that it reflects freedom's non-specific value, we believe it is more worthwhile examining whether one's definition of freedom does a better job of capturing freedom's non-specific value. Our normative criterion for judging a conception of freedom comes into play here. If freedom is something that people care about, have laid down their lives in the name of, and if a particular definition turns out on inspection to provide a definition that cannot explain why that is the case, then that

${ }^{2}$ We think he considers it partly evaluative because his measure does not include the specific value of freedom, however, nor do all other evaluative measures, for example, KlemischAhlert, 1993; Pattanaik and Xu, 2000; Bossert et al., 2003.

${ }^{3}$ Clearly, there may be other forms of non-specific value that counterbalance such a negative effect but this is not necessarily so. 
is a strong count against it as a conception of freedom. ${ }^{4}$ We believe the intention-adapted version of the negative freedom concept does indeed do a better job in this respect. If Carter and Kramer are criticizing us for not having given the argument for that claim, then they are absolutely right in doing so. That, however, was not the thrust of our paper. We tried to show that Carter's and Kramer's conceptions of freedom fail on their own account - not, yet, to give a full-fledged theory of our own.

\section{REFERENCES}

Bossert, W., P. K., Pattanaik and Y. Xu 2003. Similarity of options and the measurement of diversity. Journal of Theoretical Politics. 15: 405-422.

Carter, I. 1999. A Measure of Freedom. Oxford: Oxford University Press.

Carter, I. and M. H. Kramer 2008. How changes in one's preferences can affect one's freedom (and How they cannot): a reply to Dowding and Van Hees. Economics and Philosophy, 24: $81-96$.

Dowding, K. and M. van Hees 2007. Counterfactual success and negative freedom. Economics and Philosophy, 23: 141-162.

Hees, M. van 2000. Legal reductionism and freedom. Dordrecht: Kluwer.

Klemisch-Ahlert, M. 1993. Freedom of choice: a comparison of different rankings of opportunity sets. Social Choice and Welfare. 10: 189-207.

Kramer, M. H. 2003. The Quality of Freedom. Oxford: Oxford University Press.

Pattanaik, P. K. and Y. Xu 2000. On diversity and freedom of choice. Mathematical Social Sciences 30: 123-130.

Schwartz, B. 2004. The paradox of choice. Why more is less. New York: Harper Collins.

${ }^{4}$ It is not clear, for instance, why the sheer number of actions is a measure of freedom, rather than, say power. Carter gives a long defence of why his measure is consistent with earlier writing on negative freedom but gives not one word distinguishing his freedom function from accounts of individual power. Many of which, from Max Weber onwards, consider power to be the ability to do what one wants against the resistance or potential resistance of others. We aim to formally distinguish freedom from power which we feel is not a trivial task; and one for which our normative criterion might prove important. 